\title{
NEK9-related lethal skeletal dysplasia
}

INSERM

\section{Source}

INSERM. (1999). Orphanet: an online rare disease and orphan drug data base. NEK9related lethal skeletal dysplasia. ORPHA:464366

NEK9-related lethal skeletal dysplasia is a rare, lethal, primary bone dysplasia characterized by fetal akinesia, multiple contractures, shortening of all long bones, short, broad ribs, narrow chest and thorax, pulmonary hypoplasia and a protruding abdomen. Short bowed femurs may also be associated. 